\title{
Smectic Liquid-Crystalline Order in Suspensions of Highly Polydisperse Goethite Nanorods**
}

\author{
By Gert J. Vroege,* Dominique M. E. Thies-Weesie, Andrei V. Petukhov, Bruno J. Lemaire, and \\ Patrick Davidson
}

Colloidal dispersions are natural candidates to produce materials structured at the nanometer scale because of their propensity to self-organize into colloidal (liquid) crystals. Not only do they fall in the right size range (up to the wavelength of light necessary for photonic applications) but anisometric colloids also exhibit different length scales in different directions. Moreover, so called mineral liquid crystals ${ }^{[1]}$ are also particularly susceptible to external fields. An inherent disadvantage of colloids is their polydispersity, which is, for example, supposed to suppress the formation of smectic liquid crystals, while mineral particles also suffer from sedimentation due to a large density difference with the solvent. Their phase behavior is further complicated by size fractionation and kinetic effects. In this Communication we present a very (over $50 \%)$ polydisperse system of Goethite $(\alpha-\mathrm{FeOOH})$ nanorods where these apparent disadvantages meet to produce a wealth of concurrent phases ranging from the isotropic, nematic and columnar to-surprisingly-even a smectic phase. Our findings suggest that employing such polydisperse mineral nanorods gives access to diverse nanostructured materials, as an alternative to the route that has recently successfully employed mineralized virus suspensions. ${ }^{[2]}$

One of the paradigms in colloid science is the notion that the inherent polydispersity of colloids suppresses the formation of ordered phases. Examples are suspensions of spheres, which would refuse to crystallize above the so-called terminal

[*] Dr. G. J. Vroege, Dr. D. M. E. Thies-Weesie, Dr. A. V. Petukhov Van't Hoff Laboratory for Physical and Colloid Chemistry Utrecht University

Padualaan 8, $3584 \mathrm{CH}$ Utrecht (The Netherlands)

E-mail: g.j.vroege@chem.uu.nl

Dr. B. J. Lemaire

Centre d'Etudes techniques maritimes et fluviales

BP 60039, 60321 Compiègne (France)

Dr. P. Davidson

Laboratoire de Physique des Solides, UMR 8502 CNRS, Bat. 510

Université Paris-Sud 91405 Orsay Cedex (France)

[**] We gratefully acknowledge the help of Alex de Beer in performing our SAXS experiments, Chantal Vonk for making the TEM pictures, and Henk Lekkerkerker for general discussions. This work was performed as part of a NWO-CW TOP project of the Netherlands Organization for the Advancement of Research, which is also acknowledged for granting us the beam time. Finally, we thank the staff of the ESRF BM-26 beamline and Igor Dolbnya in particular for support. polydispersity of $5-10 \%,{ }^{[3,4]}$ and colloidal rods, which are not supposed to fit into the layers of smectic liquid crystals above about $18 \%$ length polydispersity. ${ }^{[5]}$ However, recent theories have recognized that this issue is markedly influenced by fractionation or partitioning of the colloidal components over coexisting phases.

Fractionation has been addressed for the isotropic-nematic coexistence in rod dispersions, ${ }^{[6-8]}$ in which case polydispersity does not prevent ordering since only the orientational degrees of freedom are involved. On the contrary, polydispersity even drastically widens the phase gap here since the longer rods want to orient first while shorter rods remain in the isotropic phase (this problem has only recently been addressed for truly continuous distributions $\left.{ }^{[9-12]}\right)$. For ordered systems with complete positional order the situation is more complicated, as is borne out by recent results for fractionation accompanying the crystallization of polydisperse spheres. ${ }^{[13]}$ The full incorporation of partitioning for continuous distributions now seems to redefine the concept of terminal polydispersity as pertaining to the crystalline phases only (which cannot incorporate a polydispersity of over $7 \%$ ). Theoretically, the polydisperse fluid could split off several crystalline phases and would not possess a terminal polydispersity; experimentally, it may still show up for kinetic reasons. ${ }^{[14]}$ Liquid-crystalline phases with partial positional order like smectic or columnar phases can be expected to fall in between these extremes.

Another contribution to complicating the observed phase behavior of colloids is sedimentation. Wensink and Lekkerkerker $^{[15]}$ showed that multiple (isotropic gas-isotropic fluidnematic-columnar) phase equilibria in a single test tube of a polymer-platelet mixture ${ }^{[16]}$ could be explained as a result of sedimentation, which imposes a concentration gradient on the system. The phases in contact at different heights in the system then have different osmotic pressures and different compositions at which they coexist. Sedimentation therefore also couples to fractionation, as recently described in the theory by Bellier-Castella and $\mathrm{Xu}$ for polydisperse van der Waals fluids. ${ }^{[17]}$

Here, we apply these theoretical concepts to understand the remarkably rich phase behavior of Goethite dispersions in water. Although Goethite was already used about 30000 years ago as a dark-yellow ochre pigment in paleolithic cave paintings and its special magneto-optical properties were recognized in 1902 by Majorana, ${ }^{[18]}$ only the recent work by Lemaire et al. ${ }^{[19-23]}$ revealed the special phase behavior of its 
aqueous dispersions. Their use of thin samples demonstrated that isotropic dispersions of its elongated crystallites transform into nematic and columnar liquid crystals upon increasing the concentration.

Goethite particles were synthesized as described by Lemaire et al. ${ }^{[20]}$ leading to the results shown in Figure 1. This highly polydisperse system gave interesting results in capillaries stored vertically and filled up to a height of $5 \mathrm{~cm}$
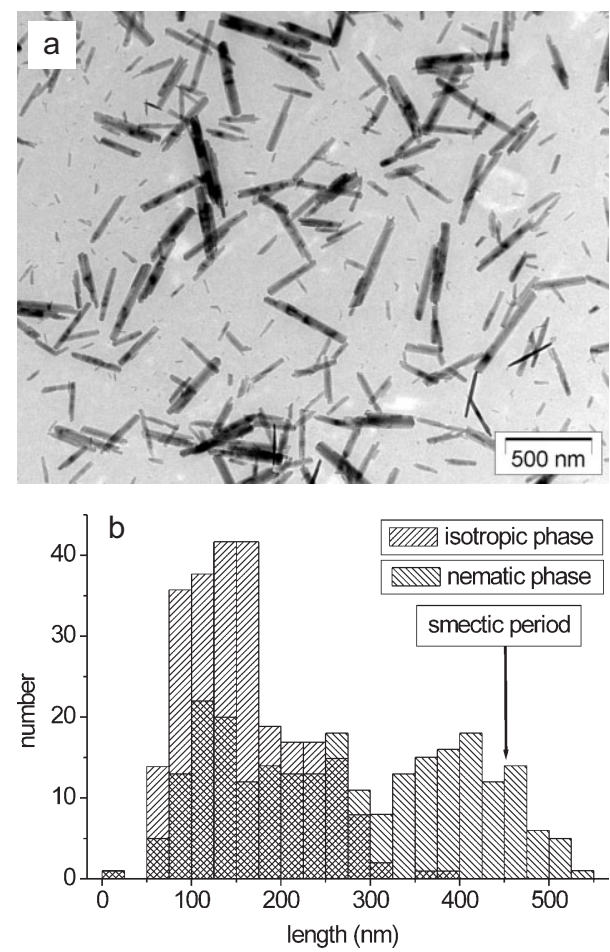

Figure 1. Goethite rods as synthesized by adding $1 \mathrm{M} \mathrm{NaOH}$ to an aqueous $0.1 \mathrm{M}$ iron nitrate solution until reaching $\mathrm{pH}^{11-12}$. After ageing for nine days the sediment was charged with $3 \mathrm{M} \mathrm{HNO}_{3}$ and three times centrifuged and redispersed in water at $\mathrm{pH} 3$. The charge density is around $0.2 \mathrm{C} \mathrm{m}^{-1}$ and the electric double-layer thickness less than $10 \mathrm{~nm}$. a) Transmission electron microscopy image of the Goethite crystallites formed with dimensions length $L=216 \mathrm{~nm}( \pm 55 \%)$, width $W=35 \mathrm{~nm}$ ( $\pm 48 \%$ ), and thickness $T \approx 16 \mathrm{~nm}$; b) length distribution in the coexisting isotropic and nematic phases. The arrow indicates the smectic periodicity.

( $0.2 \mathrm{~mm} \times 4 \mathrm{~mm}$ cross section). Above $10 \mathrm{vol} \%$ the system readily showed within a day an isotropic-nematic phase separation, where polarization microscopy showed vivid Brownian fluctuations and settling nematic tactoids smoothly coalescing with the growing nematic layer characterized by typical Schlieren patterns (Fig. 2a). Analysis of samples taken from the different layers revealed strong size partitioning between the phases (see Fig. 1b). Only on a time scale of months did a sedimentation profile become visible at the top of the isotropic phase (otherwise hardly observable, because of the intense ochreous color of the dispersion), which is in agreement with a typical sedimentation length $l_{\mathrm{g}}$ of $0.8 \mathrm{~mm}$. The distance over which an ideal barometric profile of Goethite particles of

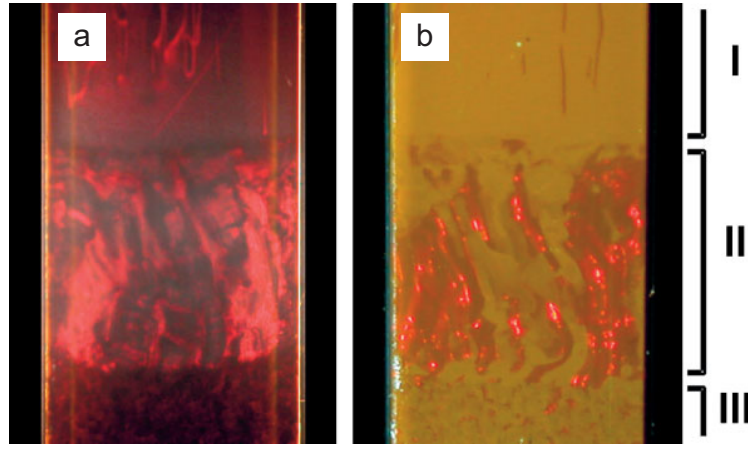

Figure 2. These photographs of a capillary with the phase-separated Goethite dispersion ( $16 \%$ volume fraction) display three distinctly different regions (apart from an isotropic layer higher up in the capillary-not shown here). a) Between crossed polarizers: Schlieren patterns typical for a nematic phase are observed in region I. b) In reflected light: brightred Bragg reflections from a smectic phase are seen in region II. The columnar phase (predominant in region III) is not distinguished by special optical characteristics.

average length $\bar{L}$, width $\bar{W}$, and thickness $\bar{T}$, would decay is given by

$l_{g}=\frac{k_{\mathrm{B}} T}{g \Delta \rho \bar{L} \bar{W} \bar{T}}$

where $k_{\mathrm{B}} T$ is the thermal energy, $\Delta \rho$ the mass density difference of Goethite and water, and $g$ the gravitational acceleration.

We prepared a range of capillaries with increasing volume fraction, but we focus here on samples of $16 \mathrm{vol} \%$. After a few days a darker layer of grainy appearance developed at the bottom of this capillary, followed after a week by the development of small red reflecting specks at the interface between the bottom layer and the nematic phase. Over a month, these specks grew into a layer at the expense of the grainy bottom layer and reached a height of about $5 \mathrm{~mm}$, showing bright red Bragg reflections in specific directions (see Fig. 2b). These observations strongly suggest the development of repeat distances within this layer on the order of the wavelength of visible light, possibly a smectic state.

To give decisive answers about the structure of the bottom layers, we used the recently developed high-resolution smallangle X-ray scattering (SAXS) setup ${ }^{[24]}$ of the Dutch-Belgian beamline BM-26B at the ESRF (Grenoble, France) and scanned the capillary. In the top layer we observe an isotropic scattering pattern; going down we see the signature of the nematic phase in Figure 3a, while the red Bragg reflections are confirmed in Figure $3 \mathrm{~b}$ as belonging to a simple smectic-A phase, with two reflection orders (sometimes even three orders, as shown in Fig. 3d) due to the interlayer spacing and a liquidlike peak parallel to the layers. The typical repeat distance is $450 \mathrm{~nm}( \pm 10 \mathrm{~nm}$ at different places in the layer), almost twice the average particle length, which points to substantial fractionation favoring the longer particles (see 

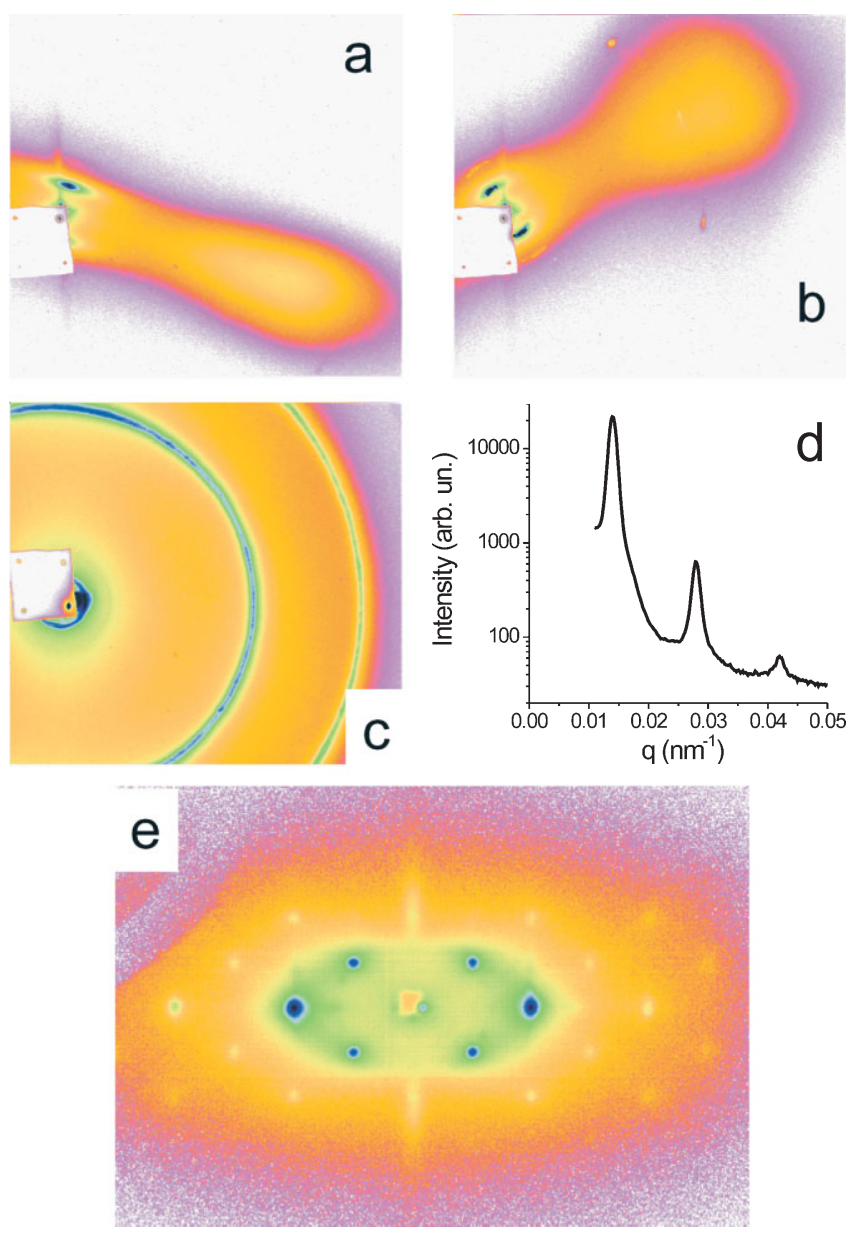

Figure 3. SAXS patterns taken with a high-resolution charge-coupled device camera from a four-phase Goethite dispersion. a) Nematic with a small-angle diffuse-scattering peak showing length correlations and a perpendicular diffuse liquidlike peak; b) smectic A with sharp first- and second-order small-angle reflections and perpendicular diffuse liquidlike scattering; c) powderlike rings of the columnar phase near the bottom of the capillary (and a remaining small-angle smectic ring); d) intensity profile along the orientation axis in the smectic $A$ phase (further down in the sample compared to (b)); e) a 2D columnar structure formed perpendicular to a horizontal $650 \mathrm{mT}$ magnetic field applied to the pattern of (b) (obtained with a lower-resolution, wider-view, gas-filled detector).

Fig. 1b). Most smectic and also many nematic patterns showed additional diffraction spots corresponding to typical distances of $56 \pm 3$ and $36 \pm 2 \mathrm{~nm}$, characteristic of the columnar phase (reflections (11) and (20) of the $c 2 \mathrm{~mm}$ symmetry group) reported before by Lemaire et al. ${ }^{[22]}$ The lower $4 \mathrm{~mm}$ of the capillary shows a powder pattern of the columnar phase (see Fig. 3c), indicating that the columnar domains are generally much smaller than the nematic and smectic domains encountered higher up in the sample.

It is striking that, at the same height where the smectic Bragg reflections are detected, indications of a columnar phase also appear. In this respect a virtually pure smectic SAXS pattern, as seen in Figure 3b, is exceptional and almost always a mixed smectic-columnar SAXS pattern is observed.
This could well be considered a kinetic effect but one could also speculate that this forms a mechanism to accommodate any remaining length polydispersity by expelling ill-fitting particles from the smectic to the columnar phase, since the latter is not disturbed by differences in particle length.

Lemaire et al. ${ }^{[19]}$ reported that small Goethite particles possess a considerable permanent magnetic moment along their long axis, presumably due to partially uncompensated spins within their antiferromagnetic crystal structure, in combination with an induced moment largest along one of the shorter axes. At small magnetic fields the permanent moment dominates, but at fields above $250 \mathrm{mT}$ particles increasingly turn perpendicular to the field. Here, we put our sample in the $650 \mathrm{mT}$ field of a small permanent magnet and observed an immediate transformation of the smectic-columnar region into a columnar structure oriented perpendicular to the field (see Fig. 3e). The scattering pattern shows a $2 \mathrm{D}$ rectangular pattern of diffraction spots typical for a distorted hexagonal columnar structure. ${ }^{[22]}$ Especially along the field many reflections (up to the fifth order) are visible. The much faster decay of the intensity of diffraction spots perpendicular to the field indicates that the 2D structure is formed in all perpendicular directions and the diffraction spots observed are actually cross sections through scattering rings around the field direction. After removal of the sample from the magnet, we again observed the typical red Bragg reflections, which indicates that the system transforms back to a smectic state.

In conclusion, aqueous dispersions of Goethite rods not only form isotropic, nematic, and columnar phases but also a newly found smectic A phase. Although smectic phases have been found before in colloidal dispersions of rodlike particles (for examples see the literature ${ }^{[25-28]}$ ), the smectic phase of Goethite is first identified in an extremely (over 50\%) polydisperse system, much higher than the terminal polydispersity of $18 \%$ determined by computer simulation of spherocylinders. ${ }^{[5]}$ It seems very likely that the fractionation and sedimentation-induced macroscopic separation associated with the isotropic-nematic phase transition help to create the circumstances to form a smectic phase within one week. However, it remains remarkable that only a very narrow range of smectic layer spacings is found. Particle lengths that do not fit seem to go to the columnar phase. A second scenario, where individual particles slowly sediment and fractionate and only then phase separate, also seems to be at work, since samples with much lower volume fractions also develop Bragg reflections after about half a year. In both scenarios, the phase development is clearly kinetically helped by the Brownian motion enabled by the presence of an electric double layer around these charged particles.

Finally, we hope to have given evidence that particle polydispersity is not always a nuisance but can at times also be very helpful - especially in combination with sedimentationand may even be employed to form new phases. Moreover, the special magnetic properties of these mineral liquid crystals give the possibility to exploit the combined effect of field response and the strong correlations present in liquid-crystalline 
phases. This allows manipulating the structures formed by applying external fields (e.g., switching between smectic and columnar phases), so that they might find applications as functional materials in the future. Our findings also suggest that employing polydisperse mineral nanoparticles in some instances forms an alternative route to produce nanomaterials instead of using suspensions of viruses, ${ }^{[2]}$ which are intrinsically monodisperse but often require painstaking experimental procedures.

Received: May 22, 2006

Final version: July 6, 2006 Published online: September 14, 2006

[1] P. Davidson, J. C. P. Gabriel, Curr. Opin. Colloid Interface Sci. 2005, 9, 377.

[2] C. E. Flynn, S.-W. Lee, B. R. Peelle, A. M. Belcher, Acta Mater. $\mathbf{2 0 0 3}, 51,5867$, and references therein.

[3] P. G. Bolhuis, D. A. Kofke, Phys. Rev. E 1996, 54, 634.

[4] P. Bartlett, P. B. Warren, Phys. Rev. Lett. 1999, 82, 1979.

[5] M. A. Bates, D. Frenkel, J. Chem. Phys. 1998, 109, 6193.

[6] L. Onsager, Ann. N. Y. Acad. Sci. 1949, 51, 627.

[7] H. N. W. Lekkerkerker, P. Coulon, R. van der Hagen, R. Deblieck, J. Chem. Phys. 1984, 80, 3427.

[8] T. Odijk, H. N. W. Lekkerkerker, J. Phys. Chem. 1985, 89, 2090.

[9] A. Speranza, P. Sollich, Phys. Rev. E 2003, 67, 061702.

[10] A. Speranza, P. Sollich, J. Chem. Phys. 2003, 118, 5213.

[11] A. Speranza, P. Sollich, J. Chem. Phys. 2002, 117, 5421.
[12] H. H. Wensink, G. J. Vroege, J. Chem. Phys. 2003, 119, 6868.

[13] M. Fasolo, P. Sollich, Phys. Rev. Lett. 2003, 91, 068301.

[14] P. N. Pusey, in Colloidal Suspensions (Eds: J. P. Hansen, D. Levesque, J. Zinn-Justin), North Holland, Amsterdam 1991, Ch. 10.

[15] H. H. Wensink, H. N. W. Lekkerkerker, Europhys. Lett. 2004, 66, 125.

[16] F. M. van der Kooij, M. Vogel, H. N. W. Lekkerkerker, Phys. Rev. E 2000, 62, 5397.

[17] L. Bellier-Castella, H. Xu, J. Phys. Condens. Matter 2003, 15, 5417.

[18] Q. Majorana, Rend. Accad. Lincei 1902, 11-1, 374.

[19] B. J. Lemaire, P. Davidson, J. Ferré, J. P. Jamet, P. Panine, I. Dozov, J. P. Jolivet, Phys. Rev. Lett. 2002, 88, 125507.

[20] B. J. Lemaire, P. Davidson, J. Ferré, J. P. Jamet, D. Petermann, P. Panine, I. Dozov, J. P. Jolivet, Eur. Phys. J. E 2004, 13, 291.

[21] B. J. Lemaire, P. Davidson, J. Ferré, J. P. Jamet, D. Petermann, P. Panine, I. Dozov, D. Stoenescu, J. P. Jolivet, Faraday Discuss. 2005, 128, 271.

[22] B. J. Lemaire, P. Davidson, P. Panine, J. P. Jolivet, Phys. Rev. Lett. 2004, 93, 267801.

[23] B. J. Lemaire, P. Davidson, D. Petermann, P. Panine, I. Dozov, D. Stoenescu, J. P. Jolivet, Eur. Phys. J. E 2004, 13, 309.

[24] A. V. Petukhov, I. P. Dolbnya, D. Aarts, G. J. Vroege, Phys. Rev. E 2004, 69, 031405.

[25] X. Wen, R. B. Meyer, D. L. D. Caspar, Phys. Rev. Lett. 1989, 63, 2760.

[26] Z. Dogic, S. Fraden, Philos. Trans. R. Soc. London, Ser. A 2001, 359, 997.

[27] H. Maeda, Y. Maeda, Phys. Rev. Lett. 2003, 90, 018303.

[28] H. Maeda, Y. Maeda, J. Chem. Phys. 2004, 121, 12655. 